\title{
PERTURBING ALMOST PERIODIC DIFFERENTIAL EQUATIONS ${ }^{1}$
}

\author{
BY SHUI-NEE CHOW
}

Communicated by Fred Brauer, September 2, 1969

Abstract. When the zero solution of an almost periodic differential system is uniform-asymptotically stable, it is shown that the zero solution is diminishingly asymptotically stable and totally asymptotically stable. Generalization of the above theorem to bounded solutions and functional differential equations with finite time-lag are obtained by the same method. The techniques used in the proof also give a more general approach to perturbation theory of differential equations.

1. Consider a system of differential equations

$$
x^{\prime}=f(t, x)
$$

and its perturbed equations

$$
x^{\prime}=f(t, x)+p(t)
$$

We always assume at least that:

(i) $f(t, x)$ is defined on $[0, \infty) \times S_{c}$, where $S_{c}=\left\{x \in R^{d}:|x|<c\right.$, $c>0\}$, such that $f(\cdot, x)$ is measurable for each $x, f(t, \cdot)$ is continuous for each $t$, and $f$ is bounded on every compact subset of $[0, \infty) \times S_{c}$;

(ii) $p(t)$ is defined and measurable on $[0, \infty)$.

In a recent paper Strauss and Yorke [2] have shown (for definitions, see $\$ 4$ )

THEOREM 1. Suppose the origin 0 is uniform-asymptotically stable (UAS) for (E), and $f$ belongs to the class of Lipschitz or linear or innerproduct or periodic functions. Then for every absolutely diminishing $p(t)$, the origin 0 is eventually uniform-asymptotically stable (EvUAS) for $(\mathrm{P})$.

For a detailed discussion of Theorem 1, refer to [2].

A natural question is: does this theorem hold if $f(t, x)$ is almost periodic? An answer is given in Theorem 2.

2. Define $f_{\tau}(t, x)=f(t+\tau, x)$ and $H(f)=\left\{f_{\tau}: \tau \in R\right\} . \bar{H}(f)$ denotes the closure of $H(f)$ in the sense of uniform convergence on all of $R \times S$, for any compact subset $S \subset S_{c}$.

AMS Subject Classifications. Primary 3451, 3453; Secondary 3445.

Key Words and Phrases. Uniform-asymptotic stability, almost periodic differential equation, closure, hull, integral asymptotic stability, total asymptotic stability.

1 Research partially supported by National Science Foundation Grant GP-8914. 
Definition. A continuous function $f: R \times S_{c} \rightarrow R^{d}$ is said to be almost periodic in $t$ uniformly in $x$ if for all $\epsilon>0$, for all compact subsets $S \subset S_{c}$, there exists $k=k(\epsilon, S)>0$, such that for every interval $(a, a+k)$, there exists $\tau \in(a, a+k)$ such that

$$
\left|f_{\tau}(t, x)-f(t, x)\right|<\epsilon, \quad \text { for all } t \in R, x \in S .
$$

BOCHNER's THEOREM. $f$ is almost periodic in $t$ uniformly in $x$ on $R \times S_{c}$ if and only if $\bar{H}(f)$ is compact.

Theorem 2. Suppose the origin 0 is UAS for $(\mathrm{E}), \bar{H}(f)$ is compact, and for every $g \in \bar{H}(f)$ solutions of $x^{\prime}=g$ are unique to the right. Then $x=0$ is integrally asymptotically stable for (E). This implies [1] for every absolutely diminishing $p(t)$, the origin 0 is EvUAS for $(\mathrm{P})$.

Outline of The PRoOF of Theorem 2. Given $\eta>0, x \in S_{c}, g \in H(f)$, $t \in[0, \infty)$, let $R(\eta ; x, g, t)$ be a set in $S_{c} \times H$, which consists of all the points $\left(\phi(t), g_{t}\right)$ for which $\phi(\cdot)$ is an absolutely continuous function defined on $[0, \infty)$,

$$
\int_{0}^{\infty}\left|\phi^{\prime}(s)-g(s, \phi(s))\right| d s<\eta \text { and } \phi(0)=x .
$$

It is not difficult to show that if for every $\epsilon>0$, there exist $\delta>0, \eta>0$ such that $|x|<\delta$ implies $R(\eta ; x, g, t) \subset S(0 \times H, \epsilon)$, for all $t \geqq 0$, $g \in \bar{H}(f)$, then the origin 0 is integrally stable for $x^{\prime}=f$. Further, the origin 0 is integrally attracting for $x^{\prime}=f$ if there exists $\delta_{0}>0$, and for every $\epsilon>0$, there exist $T>0, \eta>0$ such that $|x|<\delta_{0}$ implies $R(\eta ; x, g, t)$ $C S(0 \times H, \epsilon)$, for all $t \geqq T, g \in H(f)$. $(S(0 \times H, \epsilon)$ denotes the $\epsilon$-ball containing the set $0 \times H$ in the metric space $R^{d} \times H$.) Theorem 2 then follows from Lemma 1 and the following relation:

$$
R\left(\eta ; x, g, t+t^{\prime}\right) \subset R\left(\eta ; R(\eta ; x, g, t), t^{\prime}\right) .
$$

Lemma 1. Suppose $\bar{H}(f)$ is compact, and for every $g \in \bar{H}(f)$, solutions of $x^{\prime}=g$ are unique to the right. Let $T>0, S \subset S_{c}$ be a compact subset and $c^{\prime}<c$. Assume that $F(t, x, g) \in S_{c^{\prime}}$ for every $g \in \bar{H}(f), x \in S$, and $t \in[0, T]$. Then for every $\epsilon>0$, there exists $\eta(\epsilon)>0$ such that

$$
\int_{0}^{T}|p(t)| d t<\eta \quad \text { implies }|F(t, x, g)-F(t, x, g+p)|<\epsilon
$$

for all $t \in[0, T]$ and $g \in H(f)$, where $F(t, x, g)$ denotes the solution of $x^{\prime}=g$ through $(0, x)$.

By using a similar method, we obtain 
THEOREM 3. Under the assumptions of Theorem 2, the origin 0 is totally asymptotically stable for (E).

3. There are examples due to J. Kato and J. Yorke in which $\bar{H}(f)$ is compact, $f$ is locally Lipschitz, the origin 0 is UAS for (E), and the origin 0 is not totally stable. The reason is that there are functions $g \in \bar{H}(f)$ for which the uniqueness requirement is not met.

By using a different topology on $\bar{H}(f)$, we can obtain a different class of functions for which the conclusions of Theorems 2 and 3 hold. For example, if we use the compact-open topology, Theorems 2 and 3 hold with $f$ satisfying the condition that $f$ is bounded and uniformly continuous, on every set $R \times S$, where $S$ is a compact subset of $S_{\mathrm{c}}$. An example given by Strauss and Yorke [2] shows that this is, in a sense, the best possible result one can obtain.

There is an analog of Theorems 2 and 3 for functional differential equations with finite lag. The author learned that recently Theorem 3 for functional differential equation with finite lag has also been obtained by T. Yoshizawa and J. Kato, independently [3].

Similar results have been obtained in certain cases of nonuniqueness.

It is not hard to show that the above theorems still hold in case the perturbation depends on $x$, that is, $p$ has the form $p(t, x)$.

Details of this paper will appear elsewhere.

4. Let $F\left(t ; t_{0}, x_{0}, f\right)$ denote a solution of (E) through the point $\left(t_{0}, x_{0}\right)$, and let $F\left(t, x_{0}, f\right)=F\left(t ; 0, x_{0}, f\right)$.

The solution $x=0$ is said to be integrally stable for $(\mathrm{E})$ if for every $\epsilon>0$, there exist $\delta(\epsilon)>0$ and $\eta(\epsilon)>0$ such that $\left|x_{0}\right|<\delta$ and

$$
\int_{t_{0}}^{\infty}|p(t)| d t<\eta
$$

imply, for all $t \geqq t_{0} \geqq 0$,

$$
\left|F\left(t ; t_{0}, x_{0}, f+p\right)\right|<\epsilon .
$$

If, in addition, there exists $\delta_{0}>0$, and corresponding to any given $\epsilon>0$, there are $T(\epsilon) \geqq 0$ and $\eta(\epsilon)>0$ such that $\left|x_{0}\right|<\delta_{0}$ and inequality (1) implies inequality (2) for $t \geqq T+t_{0}$, then $x=0$ is said to be integrally asymptotically stable for $(\mathrm{E})$. The second property alone is called integral attraction of the zero solution for (E).

If we replace (1) by

$$
|p(t)|<\eta, \quad t \geqq t_{0}
$$


then we have the definition of $x=0$ being totally stable (respectively totally asymptotically stable) for (E).

The origin 0 is said to be EvUAS for (E) if there exist $\delta_{0}>0$ and $\alpha_{0} \geqq 0$, and for every $\epsilon>0$, there exist $\alpha(\epsilon)>0, \delta(\epsilon)>0$, and $T(\epsilon)>0$ such that $\left|x_{0}\right|<\delta, t \geqq t_{0} \geqq \alpha(\epsilon)$ implies $\left|F\left(t, t_{0}, x_{0}, f\right)\right|<\epsilon$ and $\left|x_{0}\right|<\delta_{0}$, $t_{0} \geqq \alpha_{0}, t \geqq T+t_{0}$ implies $\left|F\left(t ; r_{0}, x_{0}, f\right)\right|<\epsilon$.

A measurable function $p:[0, \infty) \rightarrow R^{d}$ is said to be absolutely diminishing if

$$
\int_{t}^{t+1}|p(s)| d s \rightarrow 0 \quad \text { as } t \rightarrow \infty
$$

\section{REFERENCES}

1. S. N. Chow, Remarks on integral stability (to appear).

2. A. Strauss and J. Yorke, Perturbing uniform asymptotically stable nonlinear systems, J. Differential Equations 6 (1969), 452-483.

3. T. Yoshizawa and J. Kato, $A$ relationship between uniformly asymptotic stability and total stability, Funkcial Ekvac. (to appear).

University of Maryland, College Park, Maryland 20742 